\title{
Four steps to avoid a synthetic-biology disaster
}

\section{Assess the ecological risks of synthetic microbes before they escape the lab, say Genya V. Dana, Todd Kuiken, David Rejeski and Allison A. Snow.}

$\mathrm{P}$ icture vast expanses of land, perhaps in the desert or near the sea, where giant ponds of synthetic microalgae pump out biofuels. Imagine seeds coated with engineered Escherichia coli bacteria that induce lateral root growth to reduce soil erosion, the goal of the Project Auxin team in the iGEM competition (see http://2011.igem.org).

These may sound like amazing innovations that have no downside, but what if some of the modified organisms persist and spread, despite efforts to control them? Could they disrupt the normal functions of ecosystems by transferring their altered DNA to other microbes? Might they, for instance, increase competition for resources, or disrupt crucial ecological functions?

No one yet understands the risks that synthetic organisms pose to the environment, what kinds of information are needed to support rigorous assessments, or who should collect such data. These questions have been raised before, with genetically modified seeds, for example. But unlike transgenic crops, synthetic microbes will be altered in more sophisticated and fundamental ways (such as elimination of metabolic pathways), making them potentially more difficult to regulate, manage and monitor. They might also have environmental impacts that are difficult to predict.

In July 2011, the Synthetic Biology Project at the Woodrow Wilson International Center for Scholars in Washington DC (including us) assembled a group of synthetic biologists and ecologists to explore the possible risks of introducing novel organisms into the environment, and how to assess these risks (see go.nature.com/pncgxs). These scientists are developing an eco-risk research agenda - the first of its kind for this emerging field - to help move the field forward in a productive fashion, while aiming to avoid serious ecological impacts.

It is urgent that we start this work now. Synthetic biology has already moved out of the lab, propelled by significant public and private investments in organisms modified to produce chemicals, medicines and biofuels. Researchers estimate that the global market for synthetic biology was US $\$ 1.1$ billion in 2010 . This is expected to increase to $\$ 10.8$ billion in 2016, with chemicals and energy constituting the largest share ${ }^{1}$. Such a prediction conjures images of a world of 'living factories' filled with synthetic microbes made up of modular genetic parts. It also raises the possibility that these synthetic microorganisms could escape into the environment, perhaps in large quantities.

\section{RESEARCHING RISK}

We propose four areas of risk research that scientists, in coordination with regulatory agencies and other key stakeholders, should address immediately to understand the potential ecological effects of synthetic organisms, and to regulate the use of these organisms properly.

First, differences in the physiology of natural and synthetic organisms will affect how they interact with the surrounding environment. Research in this area could, for instance, look for changes in a synthetic organism's production of toxic substances or other harmful metabolites.

Second, escaped microorganisms have the potential to survive in receiving environments (for years if in a dormant state) and to compete successfully with non-modified counterparts. Researchers will need to consider how such microbes might alter habitats, food webs or biodiversity.

Third, synthetic organisms might evolve and adapt quickly, perhaps filling new ecological niches. It is important to ascertain the rate at which the synthetic organism and its genetic material evolves so as to determine whether the organism could persist, spread or alter its behaviour in natural environments - even those that are seemingly inhospitable.

The fourth area of risk research should focus on gene transfer. Microorganisms are known for their ability to exchange genetic material with other organisms or to take
D NATURE.COM US implementation of synthetic-biology policies is slow: go.nature.com/28aqj7 up free DNA from the environment. Understanding this process is important for determining whether synthetic organisms will pass on properties such as antibiotic resistance, which could pose threats to human health.

Risk-research programmes to cover these four areas will need to provide dedicated, long-term funding to obtain high-quality data, because some experiments can take years - for example, investigating rates of evolution and the persistence of synthetic organisms and their DNA in the environment. Over time, experts can use these data to develop a model that looks at the potential impacts of synthetic biology from a lifecycle perspective $^{2}$.

Public agencies must link basic and environmental risk research by co-funding projects and requiring grant recipients to work with environmental scientists from the start. Given the complexity of the research questions, the economic and social value of success-

ful synthetic-biology applications and the potential impact of errors, we think that a minimal investment of $\$ 20$ million to $\$ 30$ million over 10 years is appropriate.

Once released, synthetic organisms cannot be retrieved. It is imperative that funding and research communities take action to prevent future ecological disasters.

Genya V. Dana, Todd Kuiken and David Rejeski are at the Woodrow Wilson International Center for Scholars, Washington DC 20004, USA. Allison A. Snow is at Ohio State University, Columbus, Ohio 43210, USA.

e-mail:gvdana@gmail.com

1. Bergin, J. Synthetic Biology: Emerging Global Markets (BCC Research, 2011); available at http://go.nature.com/wrchta

2. Davis, J. M. Comprehensive Environmental Assessment (US Environmental Protection Agency, 2010); available at http://go.nature.com/ zkardl

This article was written before G.V.D. was employed by the US Department of State. Views expressed are the author's own and do not necessarily reflect those of the US Department of State or the US government 